\title{
Erratum to: Laparoendoscopic rendezvous reduces perioperative morbidity and risk of pancreatitis
}

\author{
Alberto Arezzo • Nereo Vettoretto • \\ Federico Famiglietti - Lorenzo Moja • \\ Mario Morino
}

Published online: 1 January 2014

(C) Springer Science+Business Media New York 2013

Erratum to: Surg Endosc (2013) 27:1055-1060

DOI 10.1007/s00464-012-2562-3

The affiliation for Dr Lorenzo Moja was incompletely listed as Department of Biomedical Sciences for Health, University of Milan, Milan, Italy.

The complete affiliation is Department of Biomedical Sciences for Health, University of Milan, Milan, Italy; IRCCS Galeazzi Orthopedic Institute, Milan, Italy.

The online version of the original article can be found under doi:10.1007/s00464-012-2562-3.

\footnotetext{
A. Arezzo $(\bowtie) \cdot$ F. Famiglietti · M. Morino

Digestive, Colorectal, Oncologic, and Minimal Invasive Surgery,

University of Turin, Corso Dogliotti 14, 10126 Turin, Italy

e-mail: alberto.arezzo@mac.com; alberto.arezzo@unito.it

\section{N. Vettoretto}

Laparoscopic Surgical Unit, M. Mellini Hospital, Chiari, BS,

Italy

L. Moja

Department of Biomedical Sciences for Health,

University of Milan, Milan, Italy

L. Moja

IRCCS Galeazzi Orthopedic Institute, Milan, Italy
} 